\title{
Dermatologen trafen sich zum zweiten Mal im CityCube in Berlin
}

\author{
Ende April 2017 fand die 49. Tagung der Deutschen Dermatologischen Gesellschaft (DDG) in \\ Berlin statt. Die Vielzahl der vorgetragenen Themen und die Beteiligung zahlreicher Arbeits- \\ gemeinschaften spiegelt die im Fachbereich vorhandene Innovationskraft wider. Eine Zusammen- \\ fassung über neue Ansätze bei der Behandlung der Psoriasis, die Lasertherapie beim malignen \\ Melanom, die Fortschritte in der molekularen Diagnostik entzündlicher Erkrankungen sowie die \\ Möglichkeiten und Grenzen der Off-Label-Medikation lesen Sie im folgenden Kongressbericht.
}

\section{Lasertherapie beim Melanom?}

Bei der fraktionierten Photothermolyse (FP) erzeugen fokussierte Laserstrahlen in der Haut eine Vielzahl winziger Nekrosezonen - mikroskopisch kleine Thermozylinder mit einem Durchmesser von 70 bis $150 \mu \mathrm{m}$, sogenannte Microscopic Treatment Zones (MTZ). Das epidermale und dermale Gewebe innerhalb eines solchen MTZ ist komplett zerstört, heilt aber schnell und mit geringen Nebenwirkungen ab. Die Einsatzmöglichkeiten der noch jungen Lasermethode in der Dermatologie sind vielfältig und reichen von der Glättung gering ausgeprägter Falten über lichtgeschädigte Haut und Aknenarben bis hin zu schweren Fibrosen, traumatischen Narben, Verbrennungsnarben und chronischen Ulzera.

Wie Dr. Dieter Manstein vom Massachusetts General Hospital in Boston ausführte, verändert sich infolge der FP innerhalb von ein bis zwei Wochen die Perfusion der Mikromuskulatur, feine Nervenfasern in der Epidermis regenerieren sich. Narbiges Gewebe wird geschmeidiger und weicher, die kosmetischen Verbesserungen, die erzielt werden können, sind enorm.

\section{Verstärkende Effekte}

Doch auch in der onkologischen Dermatologie könnte die FP zukünftig eine Rolle spielen, indem synergistische Effekte der FP mit anderen Therapien ausgenutzt werden. Im Mausmodell konnte demnach die Responserate maligner Melanome auf PD-1-Inhibitoren durch FP erhöht werden. So ist bekannt, dass nur rund die Hälfte von Patienten mit fortgeschrittenen malig-

\section{"Treat-to-target" bei Psoriasis?}

Im Jahr 2008 entwickelten europäische Rheumatologen ein „treat-to-target“-Konzept für die Behandlung der rheumatoiden Arthritis (RA), das von vier übergeordneten Kriterien bestimmt wird:

- gemeinsame Therapieentscheidung von Patient und dem behandelnden Rheumatologen,

- bestmögliche Verbesserung der langfristigen gesundheitsbezogenen Lebensqualität,

- zielorientierte Therapie durch Bestimmung der Krankheits- nen Melanomen auf eine Immuncheckpointblockade mit PD-1-Inhibitoren wie Nivolumab oder Pembrolizumab anspricht. Im Laborexperiment wurden Mäuse mit BRAF/PTenmutierten Melanomen, die resistent gegenüber PD-1-Blockade waren, mit einer Tripletherapie aus fraktionierter Laserbestrahlung, topischem Imiquimod und anschließender PD-1-Blockade behandelt. Dies verlängerte gegenüber den Einzeltherapien bzw. dualen Kombinationstherapien das Überleben der Mäuse um ein Vielfaches - rund die Hälfte der erkrankten Mäuse mit bilateralen Tumoren konnte sogar geheilt werden. Ähnliche Effekte wurden bei der Behandlung von Mäusen mit Pankreaskarzinom erzeugt.

\section{Nicht allein, aber in Kombination}

Auch weiterhin, so Manstein, gelte das Prinzip: „Keine Heilung eines malignen Melanoms durch FP.“ Allerdings könne die fraktionierte Laserbestrahlung künftig womöglich eine gewichtige Rolle in der Kombinationstherapie spielen und beispielsweise die Effizienz der Immuncheckpointinhibition verstärken. Diese Option müsse nun in klinischen Studien getestet werden. Das Gewicht der Lasertherapie in der Dermatologie jedoch habe sich nach Ansicht Mansteins schon längst von der kosmetischen hin zur medizinischen Anwendung verschoben.

Kathrin von Kieseritzky

Symposium "Neue Entwicklungen in Laser und EBD“ am 27.4.2017 anlässlich der 49. Tagung der Deutschen Dermatologischen Gesellschaft in Berlin. aktivität und entsprechende Anpassung der Therapie, - Aufhalten der Gelenkentzündung [1].

Das Therapieziel sieht dabei vor, durch regelmäßiges Krankheitsmanagement bzw. kontinuierliche Kontrollen und Anpassungen bei den Patienten mit der chronischen Krankheit RA eine Remission mit geringer Krankheitsaktivität zu erreichen. Hierdurch sollen der Krankheitsprogress aufgehalten und irreversible Schäden an den Gelenken verhindert werden. 